\title{
Extracorporeal lithotripsy of pancreatic stones in patients with chronic pancreatitis and pain: A prospective follow up study
}

\author{
T Sauerbruch, J Holl, M Sackmann, G Paumgartner
}

\begin{abstract}
Extracorporeal shock wave lithotripsy of pancreatic duct stones (largest stone 12 (SD) $6 \mathrm{~mm}$ ) was performed in 24 patients with abdominal pain and a dilated duct system (main pancreatic duct 10 (3) $\mathrm{mm}$ ). The procedure was well tolerated in all but two patients; who had a mild pancreatitic attack immediately after lithotripsy. Disintegration of the stones was achieved in 21 patients. This allowed complete clearance of the duct system by an endoscopic approach in $10(42 \%)$ patients and partial clearance in $7(29 \%)$ patients. Within a mean follow up period of 24 (14) months half of the patients showed complete or considerable relief of pain and alleviation of symptoms was achieved in seven patients. Relief of pain occurred more often after complete ductal clearance. There were no fatalities within the follow up period. These findings underline the value of a combined non-surgical approach, using endoscopy and adjuvant shock wave lithotripsy to patients with large pancreatic calculi and pain attacks.
\end{abstract}

Chronic pancreatitis is often accompanied by ductal calcifications causing obstruction with subsequent progressive fibrosis of the gland. Furthermore, duct obstruction causing increased outflow pressure is believed to be one pathogenic factor underlying the persistent pain
Medical Department II, Klinikum Großhadern, Munich, Germany T Sauerbruch

J Holl

M Sackmann

G Paumgartner

Correspondence to:

Tilman Sauerbruch, MD, Medizinische

Universitätsklinik, SigmundFreud-Straße 25, 5300 BonnVenusberg, Federal Republic of Germany.

Accepted for publication 22 October 1991 associated with chronic pancreatitis. ${ }^{12}$ In fact, it has been shown that surgical decompression of the duct as well as endoscopic drainage procedures may lead to alleviation of the severe distressing pain. ${ }^{3-5}$ Endoscopic extraction of pancreatic calculi, however, may be hindered by strictures, size or location of stones. Some of these problems can be resolved by extracorporeal shock wave lithotripsy, which is tolerated without major adverse effects. ${ }^{11-14}$ In the present paper we evaluate our longterm experience with a combined non-surgical approach to patients with chronic calcifying pancreatitis by using an endoscopic access and extracorporeal lithotripsy of stones primarily not amenable to endoscopic extraction.

\section{Methods}

\section{PATIENTS}

Between 1987 and 199124 patients (20 male mean age 46 (SD 12) years) were admitted to our department with chronic pancreatitis and ductal calcifications. According to the Cambridge classification $^{15}$ retrograde pancreatography showed moderate or marked changes of the pancreatic duct system. Twelve patients had predominantly solitary stones proximal to the papilla in the main pancreatic duct and several of them also had small satellite calcifications, while the other 12 patients had multiple stones in the large pancreatic ducts. The largest stone measured $12(6) \mathrm{mm}$. The aetiology of the pancreatitis was related to alcohol in 15 patients, non-alcoholic in five and unclear in four patients. Most of the patients had a decreased exocrine pancreatic function test (tubeless pancreolauryl or fluoresceine dilaurate secretory pancreatic function tests). The mean time interval between first diagnosis of chronic calcifying pancreatitis and extracorporeal shock wave lithotropsy was 5 (5) years (median 3 years). All patients were symptomatic exhibiting the characteristic deeply seated upper abdominal pain radiating to the back. The frequency of these pain attacks ranged between several times a day and once a month. Two patients had had only one pain attack (Table I)

\section{EXTRACORPOREAL LITHOTRIPSY}

In most of the patients retrograde pancreatography and sphincterotomy of the pancreatic orifice was performed before lithotripsy. Only two patients underwent extracorporeal shock wave lithotripsy without sphincterotomy because stones or strictures prevented success- 


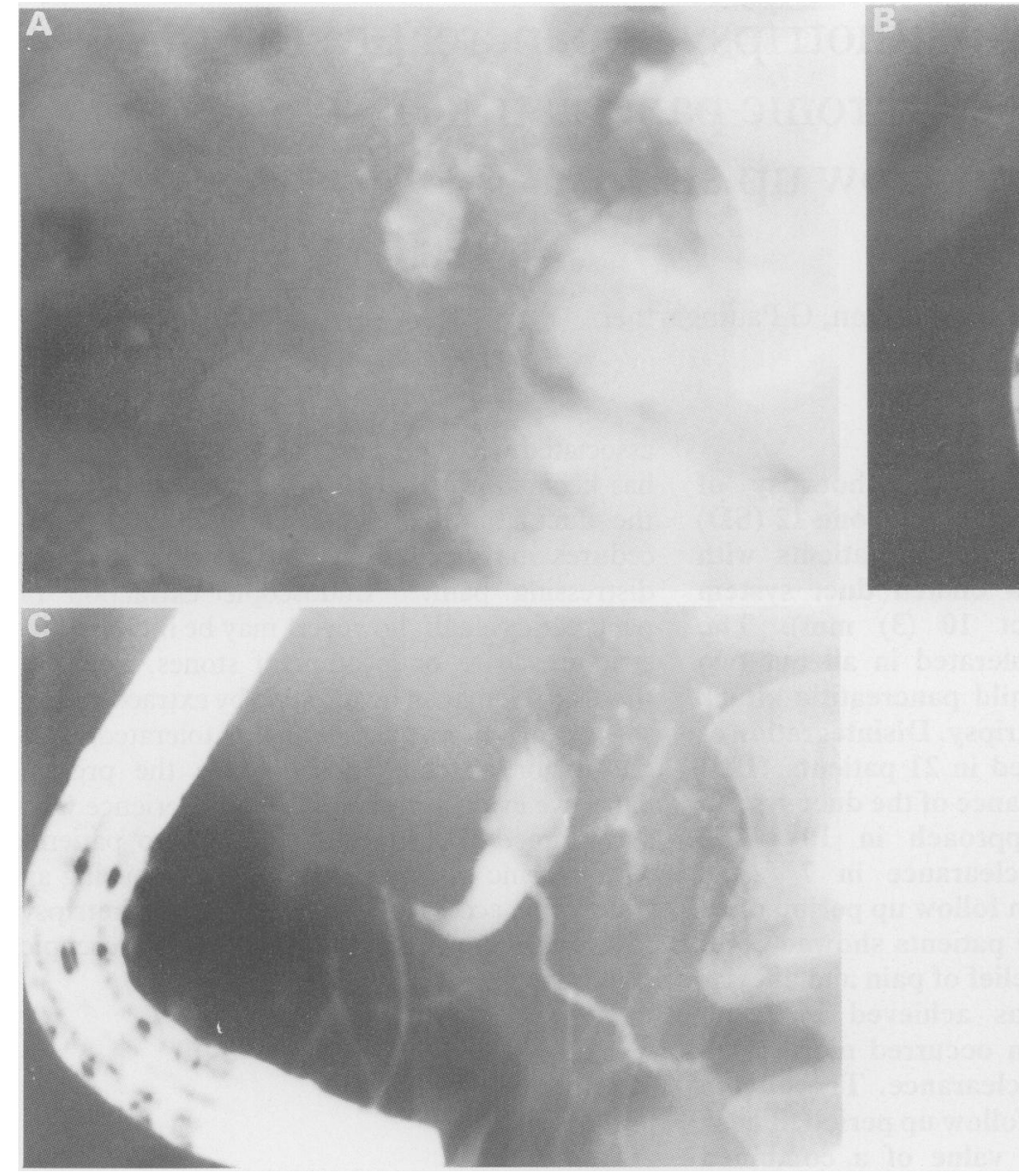

ful incision of the papilla. In these patients, attempts to open the pancreatic duct were repeated after extracorporeal shock wave lithotripsy. Extracorporeal shock wave lithotripsy was performed in supine position in a water tank lithotripter (HM 3, Dornier Medizintechnik $\mathrm{GmbH}$ ) with slight elevation of the left shoulder. ${ }^{12}$ Shock wave generation was achieved by spark gap discharge $(18-24 \mathrm{kV}, 60 \mathrm{nF})$ or electromagnetically $(11-15 \mathrm{kV})$. The stones were located into the focus of the lithotripter by fluoroscopy of the plain abdomen using a two dimensional radiography system. Two patients had in addition a nasopancreatic tube during the procedure. Eighteen patients were treated in one session, three patients in two sessions, and three in three or more sessions. On average a total of 1780 (880) shock wave pulses was applied within 30 to 60 minutes. Treatment was performed under general anaesthesia or by using intravenous analgesia with alfentanil.

\section{FOLLOW UP}

The mean follow up time was 24 (14) months. During that time patients were seen in the outpatient department or readmitted if necessary. At the end of the study all patients received a questionnaire and a telephone call to assess the current status of their symptoms. All patients responded. Relief of pain was defined as the complete alleviation of symptoms; considerable improvement, as reduction in the frequency of attacks from daily to no more than once monthly, requiring no analgesia; and improvement as a
Large stone in the main pancreatic duct and small satellite calcifications in the pancreatic head (a) in a patient with abdominal pain and chronic pancreatitis. Plain abdominal

film before $(a)$, and after extracorporeal shock wave lithotripsy (b): After endoscopic sphincterotomy and successful disintegration the main calcification had disappeared $(b)$. Retrograde pancreatogram showed complete obstruction of the main pancreatic duct before lithotripsy (c) and complete clearance of the main duct with adequate drainage after lithotripsy and endoscopic extraction of fragments $(d)$.

greater than $50 \%$ reduction, but still necessitating the use of analgesics occasionally.

\section{Results}

Fragmentation of pancreatic stones as assessed by loss of the stone contour together with reduction of the density of the calcification pattern (Figure) was achieved in 21 of 24 patients. Disintegration failed in three patients with tightly impacted stones, in whom access to the calculi was prohibited by very narrow stenoses. Stone fragmentation allowed complete clearance of the pancreatic ducts in 10 patients $(42 \%)$ and partial clearance in seven patients $(29 \%)$. Treatment failed in seven patients - that is, no clearance at all occurred, mainly because of stenoses preventing fragment extraction.

Extracorporeal shock wave lithotripsy treatment was tolerated without major adverse effects. Only two patients exhibited a mild increase in serum amylase together with pain attacks on the day after lithotripsy.

The diameter of the main pancreatic duct, as assessed by radiology (corrected in relation to the instrument), was determined in 17 patients before and after (2.4 (1.9) months) lithotripsy. It 
TABLE II Development of symptoms in relation to clearance of the pancreatic duct from calculi

\begin{tabular}{llll}
\hline & $\begin{array}{l}\text { Considerable or } \\
\text { complete relief } \\
\text { of pain }\end{array}$ & $\begin{array}{l}\text { Improvement } \\
\text { of pain }\end{array}$ & $\begin{array}{l}\text { No improvement } \\
\text { of pain }\end{array}$ \\
\hline Cuct system & 9 & 1 & - \\
Partial $(\mathrm{n}=7)$ or no clearance $(\mathrm{n}=7)$ & 3 & 7 & 4 \\
\hline
\end{tabular}

decreased (in all but one patient) on average by $22(13) \%$ from $10 \cdot 1(2 \cdot 9) \mathrm{mm}$ to $7 \cdot 8(2 \cdot 0) \mathrm{mm}$ $(\mathrm{p}<0 \cdot 0003$, paired t-test, two tailed).

Twelve patients $(50 \%)$ showed considerable or complete relief of pain. Improvement of pain attacks still requiring analgesics from time to time was achieved in a further eight patients (33\%). In four patients (17\%) symptoms were not influenced by the endoscopic sphincterotomy in conjunction with extracorporeal shock wave lithotripsy (Table II). Relief or improvement of pain occurred more often in patients with complete stone clearance than in those with partial or no clearance (Tables I, II).

Abdominal surgery was required in two patients, one with cholangitis and bile duct stenosis and a second with diffuse peritonitis and an abscess in the bursa omentalis.

The cause of this abscess remained obscure. There was no intestinal perforation or fistula from the pancreatic duct. There were no fatalities within the follow up time. Stents were placed in both the common bile duct and the pancreatic duct in two patients to obtain ductal drainage.

\section{Discussion}

Scarring of the pancreatic ductal system or calcium carbonate precipitation with stone formation can lead to obstruction of the outflow of pancreatic juice. This is believed to be one cause of recurrent pain attacks, tissue damage, and fibrosis. ${ }^{16}$ Therefore, several procedures have been performed to obtain drainage of the pancreatic duct, especially in patients with persistent and severe upper abdominal symptoms. Surgery (side-to-side pancreaticojejunostomy) has only been partially successful and mortality ranges between $0 \%$ and $10 \% .^{3-5}$ Complete or partial pain relief was observed in 40 to $100 \%$ of patients. ${ }^{3-5} 17$ Furthermore, the functional result is often insufficient because of non-simultaneous delivery of biliopancreatic secretions with food. According to one series longterm survival may be even decreased after open surgery. ${ }^{18}$ Therefore, several less invasive approaches have been evaluated, mainly endoscopic extraction of stones or stenting of pancreatic duct stenoses. ${ }^{+10}$ These approaches are combined with sphincterotomy in most patients.

In contrast with the biliary system, however, the pancreatic duct system is often too narrow for successful endoscopic manipulation and extraction of stones. Disintegration of the calculi by extracorporeal shock wave lithotripsy"1-1+ may resolve some of these technical problems. The acoustic energy necessary for successful disintegration of pancreatic stones is probably lower than that required for gall stones. Most calculi can be crashed by different lithotripters with shock wave entry from the rear ${ }^{11}{ }^{12}$ or from the ventral side. ${ }^{131+}$ Extracorporeal shock wave litho- tripsy rarely fails and if so it is rather because of tissue attenuation of the shock wave energy or tight stone impaction than stone structure or size. A more important problem is the clearance of fragments from the gland. Most patients require subsequent endoscopic extraction. This, again may be prevented in some patients by the anatomy of the altered duct system. The fragments may be located proximal to stenoses or in small peripheral ducts which preclude the passage of baskets. This explains why only $42 \%$ of our patients showed complete stone clearance. Partial clearance was achieved, however, in a further $30 \%$ of patients.

It is a matter of controversy whether abdominal pain really results from increased duct pressure caused by stone obstruction or duct stenoses. Pathogenesis of pain is generally believed to be multifactorial. In addition to duct pressure, recurrent tissue inflammation and perineural scarring are further causal factors. ${ }^{19}$ The dramatic effect of adequate drainage on abdominal symptoms, at least in some patients, however, supports the pressure hypothesis. In fact, in our series, alleviation or complete relief of symptoms was more often achieved after complete duct clearance than after partial removal of stones (Table II). There may be a substantial proportion of patients who do not respond to the drainage procedures because their symptoms are caused by other mechanisms or because duct strictures remain. A final proof of the beneficial effect of extracorporeal shock wave lithotripsy together with endoscopic procedures would be a significant difference in the outcome in a random controlled trial in order to rule out the effect of spontaneous improvement of abdominal pain attacks.

Up to now it is difficult to predict which patients will benefit from endoscopic drainage. Probably those with solitary stones in the main pancreatic duct proximal to the papilla and no severe strictures are ideal patients (Figure). Because extracorporeal shock wave lithotripsy in conjunction with endoscopic procedures is a relatively safe approach, it certainly should be attempted before surgery.

Our results are in accordance with a large experience of the Belgium group concerning the safety of extracorporeal shock wave lithotripsy and the influence on symptoms. In a series of 123 patients ${ }^{13}$ they observed complete clearance of the main pancreatic duct in $59 \%$ and complete pain relief in $45 \%$ which is similar to the $50 \%$ in our patients. Complete or partial pain relief occurred in $83 \%$ of our patients and in $85 \%$ of the Belgium patients. In the Belgium study septic events occurred more often than we observed. Influenced by our experience with lithotripsy of bile duct stones, we routinely use an antibiotic prophylaxis with the aim of lowering the incidence of post lithotripsy bacteremia.

In conclusion, extracorporeal shock wave lithotripsy has proved a safe novel therapy not only for kidney stones,,$^{20}$ gall bladder stones, ${ }^{212}$ or bile duct stones, ${ }^{2123}$ but also for pancreatic calculi. It is an effective adjunct to the nonsurgical endoscopic approach in patients with chronic calcifying pancreatitis with complete or partial relief of symptoms in around $80 \%$ of 
patients. This is comparable with the results of open surgical drainage but offers the advantage of a less invasive procedure and zero mortality to date. The complex nature of chronic pancreatitis with its difficult duct anatomy and the multifactorial pathogenesis of pain explains on the other hand a $20 \%$ failure rate with both approaches. For these reasons, the conservative treatment described here should be attempted before open surgery.

Supported by the Koerber-Foundation. The authors thank Martina Bäurer for secretarial help.

1 Ebbehoj N, Borly L, Bülow J, Rasmussen SG, Madsen P. Evaluation of pancreatic tissue fluid pressure and pain in chronic pancreatitis. A longitudinal study. Scand $\mathcal{F}$ Gastroenterol 1990; 25: 462-6.

2 Widdison AL, Alvarez C, Karanjia ND, Reber HA. Experimental evidence of beneficial effects of ductal decompression in chronic pancreatitis. Endoscopy 1991; 23: 151-4.

3 Greenlee HB, Prinz RA, Aranha GV. Long-term results of side-to-side pancreaticojejunostomy. World f Surg 1990; 14 $70-6$.

4 Cuilleret J, Guillemin G. Surgical management of chronic pancreatitis on the continent of Europe. World $\mathcal{F}$ Surg 1990 14: $11-8$.

5 Ihse I, Borch K, Larsson J. Chronic pancreatitis: Results of operations for relief of pain. World f Surg 1990; 14: 53-8.

6 Fuji T, Amano H, Harima K, Asagami F, Kinukawa K, Anyama S, TakemotoT, et al. Pancreatic sphincterotomy and pancreatic endoprosthesis. Endoscopy 1985; 90: 69-72

7 Grimm H, Meyer WH, Nam VCh, Soehendra N. New modalities for treating chronic pancreatitis. Endoscopy 1989; 21: $70-4$.

8 Huibregtse K, Schneider B, Vrij AA, Tytgat GNJ Endoscopic pancreatic drainage in chronic pancreatitis. Endoscopic pancreatic drainage

9 Devière J, Devaere S, Baize M, Cremer M. Endoscopic biliary drainage in chronic pancreatitis. Gastrointest Endosc 1990 36: $96-100$.
10 Cremer M, Devière J, Delhaye $M$, Baize $M$, Vandermeeren A. Stenting in severe chronic pancreatitis: results of medium term follow-up in seventy-six patients. Endoscopy 1991; 23 $171-6$

11 Sauerbruch T, Holl J, Sackmann M, Werner R, Wotzka R, Paumgartner G. Disintegration of a pancreatic duct stone with extracorporeal shock waves in a patient with chronic pancreatitis. Endoscopy 1987; 19: 207-8.

12 Sauerbruch T, Holl J, Sackmann M, Paumgartner G. Extracorporeal shock wave lithotripsy of pancreatic stones. $G u$ 1989; 30: 1406-11.

13 Delhaye $M$, Vandermeeren A, Baize $M$, Cremer $M$. Extracorporeal shock wave lithotripsy of pancreatic calculi: a new approach for the management of chronic pancreatitis. a new approach for the management
Gastroenterology 1992; 102: 610-20.

14 Neuhaus H. Fragmentation of pancreatic stones by extracorporeal shock wave lithotripsy. Endoscopy 1991; 23 $161-5$

15 Axon ATR, Classen M, Cotton PB, Cremer M, Freeny PC Lees WR. Pancreatography in chronic pancreatitis; international definition. Gut 1984; 25: 1107-12.

16 Sarles $\mathrm{H}$. Chronic calcifying pancreatitis. Scand $\mathcal{F}$ Gastroentero 1985; 20: 651-9.

17 Malfertheiner P, Büchler M. Indications for endoscopic or surgical therapy in chronic pancreatitis. Endoscopy 1991; 23. $185-90$.

18 Ammann RW, Akovbiantz A, Largiadér F, Schueler G. Course and outcome of chronic pancreatitis. Longitudinal study of a mixed medical-surgical series of 245 patients. study of a mixed medical-surg

19 Bockman DE, Büchler M, Malfertheiner P, Beger HG Analysis of nerves in chronic pancreatitis. Gastroenterolog 1988; 94: 1459-69.

20 Chaussy C, Brendel W, Schmiedt E. Extracorporeally induced destruction of kidney stopnes by shock-waves. Lancet 1980 ii: $1265-8$

21 Sauerbruch T, Delius M, Paumgartner G, et al. Fragmentation of gallstones by extracorporeal shock waves. $N E n g$ f Med 1986; 314: 818-22

22 Sackmann M, Pauletzki J, Sauerbruch T, Holl J, Schelling G, Paumgartner G. The Munich gallbladder lithotripsy study. Results of the first 5 years with 711 patients. Ann Intern Med 1991; 114: 290-6.

23 Sauerbruch T, Stern M, and the Study Group for shock-wave lithotripsy of bile duct stones. Fragmentation of bile duct stones by extracorporeal shock waves. A new approach to stones by extracorporeal shock waves. A new approach to Gastroenterology 1989; 96: 146-52. 